\title{
DEFICIÊNCIA DE MACRONUTRIENTES EM ESTÉVIA: SINTOMAS VISUAIS E EFEITOS NO CRESCIMENTO, COMPOSIÇÃO QUÍMICA E PRODUÇÃO DE ESTEVIOSÍDEO ${ }^{1}$
}

\author{
MARLEY MARICO UTUMI ${ }^{2}$, PEDRO HENRIQUE MONNERAT ${ }^{3}$, PAULO ROBERTO GOMES PEREIRA ${ }^{4}$, \\ PAULO CÉZAR REZENDE FONTES ${ }^{5}$ e VICENTE DE PAULO CAMPOS GODINHO ${ }^{6}$
}

\begin{abstract}
RESUMO - Conduziu-se experimento objetivando descrever sintomas visuais de deficiências de macronutrientes em estévia (Stevia rebaudiana) e avaliar seus efeitos no crescimento, composição química e produção de esteviosídeo. Os sintomas foram: clorose generalizada, com -N; folhas verdeescuras, com -P; folhas com clorose, bronzeamento e necrose, com -K; necrose de ápices, com -Ca; clorose e necrose foliar, em "V" invertido, com -Mg e folhas verde-pálidas e menores, com -S. As deficiências de $\mathrm{N}, \mathrm{K}$ e $\mathrm{Mg}$ reduziram o crescimento das folhas e a parte comercializável da planta, enquanto que a deficiência de $\mathrm{Mg}$ promoveu acentuada redução no desenvolvimento do sistema radicular. As deficiências de N, P, K e S diminuíram a relação entre matéria seca da parte aérea e das raízes, enquanto a deficiência de $\mathrm{Mg}$ aumentou-a. Todas as deficiências causaram a diminuição na absorção de macronutrientes, exceto a de $\mathrm{Ca}$, que reduziu somente a absorção de $\mathrm{Ca}$, e a de $\mathrm{K}$, que não alterou as absorções de $\mathrm{Mg}$ e S. A composição química dos cinco últimos pares de folhas totalmente expandidas representou bem o estado nutricional da planta. As deficiências de $\mathrm{K}$, Ca e $\mathrm{S}$ reduziram somente o teor de esteviosídeo, enquanto todas as deficiências, exceto a de P, diminuíram o conteúdo de esteviosídeo.
\end{abstract}

Termos para indexação: Stevia rebaudiana, nutrição mineral, nitrogênio, fósforo, potássio, cálcio, magnésio, enxofre.

\section{MACRONUTRIENT DEFICIENCIES IN STEVIA: \\ VISUAL SYMPTOMS AND EFFECTS ON GROWTH, CHEMICAL COMPOSITION, AND STEVIOSIDE PRODUCTION}

\begin{abstract}
This experiment was carried out to describe visual symptoms of macronutrients deficiencies in Stevia rebaudiana, and the effects of these deficiencies on growth, chemical composition and stevioside production. The symptoms were: yellowing with -N; dark green leaves with -P; clorotic, mottled, and necrotic leaves, with $-\mathrm{K}$; apical necrosis, with -Ca; leaves with clorosis and necrosis in inverted "V" shape, with -Mg, and small pale green leaves, with -S. N, K, and Mg deficiencies reduced the leaf growth, therefore the plant marketable part. $\mathrm{Mg}$ deficiency caused greater reduction on the development of the radicular system. N, P, K, and S deficiencies decreasead the shoot:root dry weigth ratio, while $\mathrm{Mg}$ deficiency increased it. All deficiencies decreased the absorption of macronutrients, except of $\mathrm{Ca}$, which reduced only the absorption of $\mathrm{Ca}$, and $\mathrm{K}$, that did not affect the absorption of $\mathrm{Mg}$ and $\mathrm{S}$. The chemical content of the last five fully expanded leaf pairs showed good correlation with the plant nutritional status. The deficiencies of $\mathrm{K}, \mathrm{Ca}$, and $\mathrm{S}$ decreased the concentration of stevioside while all deficiencies, except of $\mathrm{P}$, decreased the stevioside content.
\end{abstract}

Index terms: Stevia rebaudiana, mineral nutrition, nitrogen, phosphorus, potassium, calcium, magnesium, sulphur.

\footnotetext{
${ }^{1}$ Aceito para publicação em 26 de agosto de 1998.

Extraído da Dissertação de Mestrado, apresentada pelo primeiro autor à Universidade Federal de Viçosa (UFV).

${ }^{2}$ Eng ${ }^{\mathrm{a}}$ Agr ${ }^{\mathrm{a}}$, Dr ${ }^{\mathrm{a}}$, Embrapa-Centro de Pesquisa Agroflorestal de Rondônia (CPAF-RO), Caixa Postal 405, CEP 78995-000 Vilhena, RO. E-mail: mutumi@netview.com.br
}

\footnotetext{
${ }^{3}$ Eng. Agr., Ph.D., Prof. Convidado, Centro de Ciências Tecnológicas e Agrárias, Universidade Estadual do Norte Fluminense (UENF), CEP 28015-620 Campos dos Goytacazes, RJ

${ }^{4}$ Eng. Agr., Dr., Prof. Adjunto, Dep. de Fitotecnia, Univ. Fed. de Viçosa (UFV), CEP 36571-000 Viçosa, MG.

${ }^{5}$ Eng. Agr., Ph.D., Prof. Titular, Dep. de Fitotecnia, UFV.

${ }^{6}$ Eng. Agr., Dr., Embrapa-CPAF-RO.
} 


\section{INTRODUÇ̃̃̃O}

A estévia, Stevia rebaudiana (Bert.) Bertoni, é uma planta pertencente à família Compositae, originária dos trópicos, e no gênero Stevia, a $S$. rebaudiana tem-se destacado como produtora de edulcorantes naturais (Felippe, 1977). Os edulcorantes extraíveis da estévia são terpenos glicosídicos, denominados esteviosídeo, esteviolbiosídeo, rebaudeosídeos $\mathrm{A}, \mathrm{B}, \mathrm{C}, \mathrm{D}$ e $\mathrm{E}$ e dulcosídeo $\mathrm{A}$; dentre estes, os principais são o esteviosídeo e o rebaudeosídeo A (Alvarez, 1984).

Pode-se obter com a estévia rendimentos de 1,5 a 2,0 $\mathrm{t}$ de folhas secas/ha/ano, apresentando de 6 a 15\% de esteviosídeo (Donalísio et al., 1982).

O objetivo deste trabalho é descrever os sintomas de deficiências de macronutrientes $(\mathrm{N}, \mathrm{P}, \mathrm{K}$, $\mathrm{Ca}, \mathrm{Mg}$ e S) em estévia e determinar os efeitos desses elementos no crescimento, composição mineral e produção de esteviosídeo.

\section{MATERIAL E MÉTODOS}

Este trabalho foi conduzido em casa de vegetação, na Universidade Federal de Viçosa, no período de 18 de junho a 20 de agosto de 1991. As plantas foram obtidas a partir do enraizamento de estacas, usando-se solução nutritiva, com a seguinte composição: $5,2 \mathrm{mmol} / \mathrm{L}$ de $\mathrm{N}$ (4,6 mmol/L como $\mathrm{NO}_{3}{ }^{-}$e $0,6 \mathrm{mmol} / \mathrm{L}$ como $\mathrm{NH}_{4}{ }^{+}$); $0,4 \mathrm{mmol} / \mathrm{L}$ de $\mathrm{P} ; 2,0 \mathrm{mmol} / \mathrm{L}$ de $\mathrm{K} ; 2,0 \mathrm{mmol} / \mathrm{L}$ de $\mathrm{Ca}$; $0,6 \mathrm{mmol} / \mathrm{L}$ de $\mathrm{Mg} ; 0,6 \mathrm{mmol} / \mathrm{L}$ de $\mathrm{S} ; 35 \mu \mathrm{mol} / \mathrm{L}$ de Fe-EDTA; $25 \mu \mathrm{mol} / \mathrm{L}$ de B; $0,5 \mu \mathrm{mol} / \mathrm{L}$ de $\mathrm{Cu}$; 2,0 $\mu \mathrm{mol} / \mathrm{L}$ de $\mathrm{Mn} ; 2,0 \mu \mathrm{mol} / \mathrm{L}$ de $\mathrm{Zn} ; 0,5 \mu \mathrm{mol} / \mathrm{L}$ de $\mathrm{Mo}$ e $50 \mu \mathrm{mol} / \mathrm{L}$ de $\mathrm{Cl}$; o $\mathrm{pH}$ foi ajustado para $5,3 \pm 0,5$, no enraizamento, e 5,7 $\pm 0,5$, no crescimento, em condições similares às empregadas por Godinho et al. (1995).

O delineamento utilizado foi o de blocos casualizados, com sete tratamentos e cinco repetições. Foram mantidas duas plantas por vaso, contendo nove litros de solução nutritiva cada, com arejamento efetuado por compressor automático e tubos de plástico. Manteve-se o volume das soluções pela adição de água deionizada, sendo que as soluções foram trocadas semanalmente.

Os tratamentos foram: completo $(\mathrm{T})$, menos nitrogênio (-N), menos fósforo (-P), menos potássio (-K), menos cálcio $(-\mathrm{Ca})$, menos magnésio $(-\mathrm{Mg})$ e menos enxofre $(-S)$. Nos tratamentos com deficiências induzidas, houve omissão total do respectivo nutriente, a partir do $17^{\circ}$ dia após o transplante, exceto no tratamento $-\mathrm{Ca}$, em que se adicionou $10 \%$ da concentração de Ca do tratamento completo.

Foram realizadas observações diárias para caracterizar o aparecimento e a evolução dos sintomas de deficiência de macronutrientes.

As plantas foram colhidas no início do florescimento, 45 dias após o transplante, e divididas em raiz, caule, folhas indicadoras, constituídas pelos cinco últimos pares de folhas totalmente expandidas de cada ramo, e folhas restantes. As amostras foram secadas em estufa a $70^{\circ} \mathrm{C}$, com ventilação forçada, durante 48 horas, e pesadas. Analisaram-se os teores de $\mathrm{N}, \mathrm{P}, \mathrm{K}, \mathrm{Ca}, \mathrm{Mg}$ e $\mathrm{S}$.

Após digestão nitroperclórica e sulfúrica, determinaram-se N, pelo método de Nessler (Jackson, 1958); P, colorimetricamente, por método modificado por Braga \& Defelipo (1974); K, por fotometria de chama; $\mathrm{Ca}$ e $\mathrm{Mg}$ por espectrofotometria de absorção atômica (Association of Official Analytical Chemists, 1975) e S, por turbidimetria do sulfato (Jackson, 1958). O esteviosídeo foi determinado pelo método de Angelucci (Angelucci , 1979).

Efetuou-se a análise de variância, sendo comparadas as médias dos tratamentos com a testemunha pelo teste de Dunnett, a 5\% de probabilidade.

\section{RESULTADOS E DISCUSSÃO}

As deficiências de macronutrientes na estévia afetaram o crescimento (Tabela 1), e seus sintomas visuais foram os comumente observados na maioria das plantas (Malavolta et al., 1989). Nas condições experimentais, essas deficiências ocorreram na seguinte ordem: $\mathrm{Ca}, \mathrm{N}, \mathrm{K}, \mathrm{Mg}, \mathrm{P}$ e S.

As plantas com deficiência de $\mathrm{N}$ apresentaram clorose nas folhas basais, que progrediu para as folhas medianas e superiores e generalizou-se posteriormente em toda a planta. As ramificações apresentaram-se finas e longas. As folhas basais, que já estavam cloróticas, tornaram-se necrosadas, ocorrendo abscisão prematura. Houve redução generalizada no crescimento (Tabela 1).

Nas plantas deficientes em $\mathrm{P}$, as folhas totalmente expandidas tinham coloração verde-escura no terço médio e superior da planta, apresentando ainda curvatura dos bordos e ápices para baixo. As folhas mais novas apresentaram limbos mais estreitos. $\mathrm{O}$ sistema radicular das plantas deficientes em $\mathrm{P}$ apresentou coloração mais escura e menor quantidade de radicelas. 
A deficiência de K provocou curvatura para baixo nas folhas maduras, com cloroses internervais e nas bordas das folhas mais novas. As áreas cloróticas internervais tornaram-se bronzeadas e encarquilhadas, e a seguir surgiram pontos necróticos que coalesceram. Houve queda de folhas basais e reduzido desenvolvimento do sistema radicular.

Com deficiência de $\mathrm{Ca}$, verificou-se necrose apical dos primórdios foliares, a qual evoluiu para morte descendente típica. Ocorreram também pequenos pontos necróticos nos ápices radiculares, seguidos de escurecimento e morte. Os ramos formados apresentaram-se suscetíveis à quebra, e as raízes tiveram o diâmetro e o comprimento reduzidos.

$\mathrm{Na}$ deficiência de $\mathrm{Mg}$, as folhas ficaram cloróticas, com as nervuras verdes e as bordas e o ápice do limbo voltados para baixo. A clorose em "V" invertido progrediu para o ápice do limbo, e tornouse bronzeada e necrosada. Os ramos ficaram mais finos, e algumas folhas do terço mediano apresentaram-se retorcidas. $\mathrm{O}$ sistema radicular foi reduzido.

A deficiência de $\mathrm{S}$ provocou coloração verde pálida, reduziu o tamanho das folhas novas e curvou para baixo as folhas desenvolvidas. Houve também redução na formação de radicelas e engrossamento das raízes.

As deficiências de N, P, K e Mg reduziram o crescimento vegetativo, pela menor produção de folhas e caule. Nas plantas deficientes em $P$, não houve alteração na produção foliar. A deficiência de $\mathrm{Mg}$ também promoveu acentuada redução no desenvolvimento do sistema radicular (Tabela 1).
Observou-se a redução no teor de cada macronutriente induzido pelas suas respectivas deficiências na solução nutritiva. Esta redução ocorreu principalmente nas folhas indicadoras, isto é, nos cinco últimos pares de folhas totalmente expandidas (Tabela 2).

Todas as deficiências promoveram decréscimo na absorção de $\mathrm{Ca}$ e diminuíram a absorção dos outros macronutrientes, exceto a deficiência de $\mathrm{Ca}$, que reduziu somente a absorção de $\mathrm{Ca}$; a deficiência de $\mathrm{K}$, porém, não alterou as absorções de $\mathrm{Mg}$ e de $\mathrm{S}$ (Tabela 3).

O conteúdo de esteviosídeo nas plantas foi reduzido em todas as deficiências, exceto na de $\mathrm{P}$, que não o alterou significativamente (Tabela 4), enquanto que Lima Filho et al. (1997) verificaram que dentre as deficiências de macronutrientes, somente a de $\mathrm{Mg}$ não afetou a quantidade de esteviosídeo foliar.

Os menores conteúdos de esteviosídeos nas plantas deficientes em $\mathrm{N}$ e $\mathrm{Mg}$ ocorreram devido à diminuição da produção de matéria seca foliar (Tabela 1), ou devido à redução do teor foliar, nas deficiências de Ca e S (Tabela 4), ou ainda devido a ambos os fatores, no caso da deficiência de K (Tabelas 1 e 4). Lima Filho et al. (1997) observaram que as deficiências de N, P, K e S reduziram a quantidade de esteviosídeo produzido, em decorrência da diminuição da produção foliar. A deficiência de Ca, porém, teve efeito tanto no teor do edulcorante foliar quanto na produção de folhas.

A quantidade de esteviosídeo produzida por planta (conteúdo), depende da produção da matéria seca

TABELA 1. Produção de matéria seca (g/planta) em estévia (Stevia rebaudiana) submetida às deficiências minerais de macronutrientes, em solução nutritiva.

\begin{tabular}{llcccl}
\hline Tratamento & Raiz & Caule & Folha & Parte aérea & Total \\
\hline Completo & 1,60 & 17,91 & 13,57 & 31,49 & 33,08 \\
-N & $2,61^{*}$ & $7,33^{*}$ & $7,48^{*}$ & $14,81^{*}$ & $17,42^{*}$ \\
$-\mathrm{P}$ & $2,30^{*}$ & $13,96^{*}$ & 11,44 & $25,40^{*}$ & 27,70 \\
$-\mathrm{K}$ & 1,11 & $9,35^{*}$ & $7,79^{*}$ & $17,14^{*}$ & $18,24^{*}$ \\
$-\mathrm{Ca}$ & 1,16 & 15,03 & 12,69 & 27,72 & 28,87 \\
$-\mathrm{Mg}$ & $0,82^{*}$ & $9,61^{*}$ & $10,30^{*}$ & $19,90^{*}$ & $20,72^{*}$ \\
-S & $2,83^{*}$ & 14,71 & 12,07 & 26,77 & 29,60 \\
\hline C.V. $(\%)$ & 21,65 & 15,80 & 12,79 & 14,05 & 14,03 \\
\hline
\end{tabular}

* Difere da testemunha (completo) pelo teste de Dunnett, a 5\% de probabilidade. 
TABELA 2. Teor (dag/kg) de N, P, K, Ca, Mg e S na folha indicadora em estévia (Stevia rebaudiana) submetida às deficiências minerais de macronutrientes, em solução nutritiva.

\begin{tabular}{lllllll}
\hline Tratamento & $\mathrm{N}$ & $\mathrm{P}$ & $\mathrm{K}$ & $\mathrm{Ca}$ & $\mathrm{Mg}$ & $\mathrm{S}$ \\
\hline Completo & 3,56 & 0,74 & 4,36 & 0,52 & 0,26 & 0,26 \\
- N & $1,08^{*}$ & $0,24^{*}$ & $3,44^{*}$ & 0,46 & $0,19^{*}$ & $0,09^{*}$ \\
- P & $2,92^{*}$ & $0,10^{*}$ & $3,65^{*}$ & $0,39^{*}$ & 0,20 & $0,16^{*}$ \\
- K & $4,46^{*}$ & 0,61 & $0,52^{*}$ & $0,78^{*}$ & $0,79^{*}$ & $0,66^{*}$ \\
- Ca & $3,15^{*}$ & 0,84 & 4,59 & $0,11^{*}$ & $0,39^{*}$ & 0,26 \\
- Mg & $2,98^{*}$ & $0,54^{*}$ & $3,20^{*}$ & $0,33^{*}$ & $0,03^{*}$ & 0,31 \\
- S & $2,82^{*}$ & $0,43^{*}$ & $3,29^{*}$ & $0,42^{*}$ & 0,23 & $0,11^{*}$ \\
\hline C.V. $(\%)$ & 7,08 & 17,95 & 6,78 & 11,38 & 12,32 & 16,42 \\
\hline
\end{tabular}

* Difere da testemunha (completo) pelo teste de Dunnett, a 5\% de probabilidade.

TABELA 3. Conteúdo total (mg/planta) de $\mathrm{N}, \mathrm{P}, \mathrm{K}, \mathrm{Ca}, \mathrm{Mg}$ e $\mathrm{S}$ em estévia (Stevia rebaudiana) submetida às deficiências de macronutrientes, em solução nutritiva.

\begin{tabular}{lcccccc}
\hline Tratamento & $\mathrm{N}$ & $\mathrm{P}$ & $\mathrm{K}$ & $\mathrm{Ca}$ & $\mathrm{Mg}$ & $\mathrm{S}$ \\
\hline Completo & 676,0 & 161,4 & 1111,4 & 181,6 & 70,1 & 113,6 \\
- N & $118,4^{*}$ & $51,3^{*}$ & $488,0^{*}$ & $90,2^{*}$ & $23,9^{*}$ & $35,9^{*}$ \\
- P & $5101^{*}$ & $27,0^{*}$ & $912,4^{*}$ & $130,3^{*}$ & $35,8^{*}$ & $74,7^{*}$ \\
- K & $473,4^{*}$ & $98,8^{*}$ & $185,8^{*}$ & $141,1^{*}$ & 80,3 & 106,2 \\
- Ca & 611,0 & 195,1 & 1006,6 & $51,9^{*}$ & 73,8 & 94,6 \\
- Mg & $432,7^{*}$ & $103,8^{*}$ & $625,6^{*}$ & $107,2^{*}$ & $7,2^{*}$ & $84,6^{*}$ \\
- S & $489,6^{*}$ & $112,8^{*}$ & $915,0^{*}$ & $142,8^{*}$ & $39,5^{*}$ & $24,3^{*}$ \\
\hline C.V. $(\%)$ & 12,32 & 15,54 & 14,03 & 15,79 & 20,47 & 14,82 \\
\hline
\end{tabular}

* Difere da testemunha (completo) pelo teste de Dunnett, a $5 \%$ de probabilidade.

TABELA 4. Teor (dag/kg matéria seca foliar) e conteúdo de esteviosídeo foliar ( $\mathrm{g} / \mathrm{planta}$ ) em estévia (Stevia rebaudiana) submetida às deficiências de macronutrientes, em solução nutritiva.

\begin{tabular}{lcc}
\hline Tratamento & \multicolumn{2}{c}{ Esteviosídeo } \\
\cline { 2 - 3 } & Teor & Conteúdo \\
\hline Completo & 11,96 & 1,62 \\
- N & 9,23 & $0,69^{*}$ \\
- P & 11,03 & 1,27 \\
- K & $9,11^{*}$ & $0,70^{*}$ \\
- Ca & $8,73^{*}$ & $1,10^{*}$ \\
- Mg & 10,63 & $1,10^{*}$ \\
- S & $7,11^{*}$ & $0,86^{*}$ \\
\hline C.V. (\%) & 16,29 & 21,48 \\
\hline
\end{tabular}

* Difere da testemunha (completo) pelo teste de Dunnett, a $5 \%$ de probabilidade. foliar e do teor de esteviosídeos; portanto, qualquer condição que reduza o teor de esteviosídeo ou a produção de folhas conduzirá à diminuição do conteúdo de esteviosídeo na planta.

\section{CONCLUSÕES}

1. Os sintomas visuais apresentados pelas deficiências de macronutrientes nas plantas de estévia são os mesmos comumente verificados na maioria das plantas.

2. A composição química dos cinco últimos pares de folhas totalmente expandidas representa bem o estado nutricional da planta.

3. As deficiências de N, K e Mg promovem diminuição na produção de folhas.

4. As deficiências de $\mathrm{K}$, Ca e S reduzem o teor de esteviosídeo. 
5. As deficiências de macronutrientes, exceto a de $\mathrm{P}$, reduzem a produção de esteviosídeo.

\section{AGRADECIMENTOS}

Aos professores da Fundação Universidade Estadual de Maringá (FUEM), Mauro Alvarez e Altair Bertonha, pelo apoio e pelas mudas de estévia.

\section{REFERÊNCIAS}

ALVAREZ, M. Stevia rebaudiana (Bert.) Bertoni: estado atual de conhecimento. Maringá: FUEM, 1984. 123p. Relatório de Pesquisa.

ANGELUCCI, E. O estevióside de plantas brasileiras de Stevia rebaudiana Bertoni e a potencialidade de seu emprego em formulações hídricas e carbonatadas. São Paulo: Faculdade de Ciências Farmacêuticas, USP, 1979. 78p. Tese de Doutorado.

ASSOCIATION OF OFFICIAL ANALYTICAL CHEMISTS. Official methods of analysis. 12.ed. Washington, DC, 1975. 1094p.

BRAGA, J.M.; DEFELIPO, B.V. Determinação espectrofotométrica de fósforo em extratos de solos e plantas. Revista Ceres, Viçosa, MG, v.21, p.73-85, 1974.

DONALÍSIO, M.G.R.; DUARTE, F.R.; PINTO, A.J.; SOUZA, C.J. Estévia. Agronômico, v.34, p.65-68, 1982.

FELIPPE, G.M. Stevia rebaudiana (Bert.) Bertoni: uma revisão. Ciência e Cultura, São Paulo, v.29, n.11, p.1240-1248, 1977.

GODINHO, V. de P.C.; MONNERAT, P.H.; UTUMI, M.M.; FONTES, P.C.R.; PEREIRA, P.R.G. Sintomas visuais de deficiência de potássio, acúmulo de matéria seca e características das fibras de rami em resposta a níveis de potássio na solução nutritiva. Revista Ceres, Viçosa, MG, v.42, n.240, p.139-154, 1995.

JACKSON, M.L. Soil chemical analysis. Englewood Cliffs: Prentice-Hall, 1958. 498p.

LIMA FILHO, O.F. de; MALAVOLTA, E.; YABICO, H.Y. Influência de estresses nutricionais no teor e produção de esteviosídeo durante o desenvolvimento da estévia. Pesquisa Agropecuária Brasileira, Brasília, v.32, n.5, p.489-494, maio 1997.

MALAVOLTA, E.; VITTI, G.C.; OLIVEIRA, S.A. de. Avaliação do estado nutricional das plantas: princípios e aplicações. Piracicaba: POTAFOS, 1989. $201 \mathrm{p}$. 